\title{
Structure et productivité en gomme des peuplements à Acacia senegal (L) Willd. et Acacia seyal Del. de Massenya au Tchad
}

\author{
Received: 2016-11-24; revised: 2017-09-25; accepted: 2017-10-01
}

\author{
Élisée Mbayngone ${ }^{*}$, Serge Mélom², Ali Brahim Béchir ${ }^{3}$ et Pierre Marie Mapongmetsem² \\ ${ }^{1}$ Université de N’Djaména, Faculté des Sciences Exactes et Appliquées, Laboratoire de Botanique Systématique et \\ d'Écologie Végétale, B.P. 1027 N’Djaména/Tchad, Tél.: 23566 368664, embayngone@ymail.com \\ ${ }^{2}$ Université de N'Gaoundéré, Faculté des Sciences, B.P. 454 N'Gaoundéré/Cameroun, Tél. 235634696 21/+237 777979 \\ 96, sergemelom@yahoo.fr, piermapong@yahoo.fr \\ ${ }^{3}$ Institut National Supérieur d'Élevage de Moussoro (INSEM), Tél.: +235 662542 61, abrabechir@yahoo.fr \\ *Auteur correspondant Tel.: + 235663686 64, embayngone@ymail.com
}

\begin{abstract}
Résumé: La connaissance du potentiel et de la productivité d'une ressource est une donnée nécessaire à l'élaboration d'une bonne politique de sa gestion. La structure et la productivité des peuplements à Acacia seyal Del. et à Acacia senegal (L) Willd.ont été étudiées dans les formations naturelles de Massenya au Tchad. Sur la base de 32 placeaux de $900 \mathrm{~m}^{2}$, le diamètre et la hauteur de tous les individus d'espèces ligneuses ont été mesurés. Pour des espèces adultes à port arbustif, le diamètre est mesuré à $50 \mathrm{~cm}$ du sol. Les individus juvéniles sont simplement comptés et rangés en classe de hauteur. L'étude de la structure des peuplements et de deux espèces d'Acacia a été réalisée à travers le nombre de tiges à l'hectare et les classes de diamètre. L'Indice de Valeur d'Importance (IVI) a été utilisée pour apprécier la prédominance des espèces sur le site. La productivité des peuplements en gomme a été évaluée en fonction de la production moyenne d'un arbre qui était de $250 \mathrm{~g}$. Les peuplements à Acacia de Massenya sont très denses $\left(619 \pm 269\right.$ tiges/ha), mais à surface terrière faible $\left(7,10 \pm 1,20 \mathrm{~m}^{2} /\right.$ ha) due à un grand nombre d'individus de petit diamètre $(11,1 \pm 2,2 \mathrm{~cm})$. Ce sont des peuplements généralement arbustif (hauteur de 5,2 $\pm 0,9 \mathrm{~m}$ ) avec un bon potentiel de juvéniles (408 \pm 267 tiges/ha) pouvant se régénérer naturellement. Toutefois, Acacia seyal semble plus apte à coloniser d'autres milieux qu'Acacia senegal. Sur les sept espèces qui prédominent sur le site, Acacia seyal et Acacia senegal réalisent des IVI plus élevés (respectivement 79 et 54). Les espèces à bon potentiel de régénération sont Acacia seyal ( $65 \pm 8$ juv./ha), Acacia senegal (58 \pm 10 juv./ha) et Guiera senegalensis ( $51 \pm 8$ juv./ha). La production annuelle de gomme est estimée à $56 \pm 6 \mathrm{~kg} /$ ha de gomme friable (à Acacia seyal) et $41 \pm 5 \mathrm{~kg} /$ ha de gomme dure (à $A$. senegal).
\end{abstract}

Mots clés: Acacia senegal, Acacia seyal, structure et productivité de gomme, zone sahélo-soudanienne, Tchad

\section{Structure And gum productivity of Acacia senegal (L) Willd. ANd Acacia seyal Del. STANDS Of MASSENYA IN ChAD}

Summary: Knowledge of the potential and productivity of a resource is a particular need for the development of a good management policy. Therefore, structure and the productivity of Acacia seyal Del. and Acacia senegal (L) Willd. stands were studied in the natural vegetation of Massenya (Chad). Based on 32 plots of $900 \mathrm{~m}^{2}$, diameter $(50 \mathrm{~cm}$ above ground) and height of all trees and shrubs were measured. For shrubs, the diameter was measured at $50 \mathrm{~cm}$ from the ground. The juveniles were counted and assigned to height classes. The population structure was characterized by the number of stems per hectare and diameter classes. The Index of the Value of Importance (IVI) was used to assess the prevalence of species on the site. Stand productivity was evaluated based on the tree's mean production which was $250 \mathrm{~g}$. The stands of Massenya's Acacia are very dense $(619 \pm 269 \mathrm{stems} / \mathrm{ha})$, but have a low basal area $\left(7.1 \pm 1.2 \mathrm{~m}^{2} /\right.$ ha $)$ due to a high number of small diameter individuals $(11.1 \pm 2.2 \mathrm{~cm}$ ). In general, these are shrub populations (height $5.2 \pm 0.9 \mathrm{~m}$ ) with good potential for juveniles $(408$ \pm 267 stems / ha) that can regenerate naturally. However, Acacia seyal seems to colonize other environments than Acacia senegal. Of the seven species predominant on the site, Acacia seyal and Acacia senegal have the highest IVI (79 and 55). Species with good regeneration potential are Acacia seyal (64.58 $\pm 8.26 \mathrm{juv}$./ha) Acacia senegal (58 $\pm 10 \mathrm{juv}$./ha) and Guiera senegalensis (51 $\pm 8 \mathrm{juv} . / \mathrm{ha})$. The annual gum production is estimated at $56 \pm 6 \mathrm{~kg} / \mathrm{ha}$ of friable gum (Acacia seyal) and $41 \pm$ $5 \mathrm{~kg} / \mathrm{ha}$ of hard gum (Acacia senegal).

Key words: Acacia senegal, Acacia seyal, gum productivity, sudano-sahelian zone, Chad

\section{Struktur und Gummi-Produktivität der Bestände Von ACACIA SENEGal (L) WiLLD. UNd ACACIA SEYAL DEL. VON MASSENYA IM TSCHAD}

Zusammenfassung: Die Kenntnis des Potenzials und der Produktivität einer Ressource ist eine wichtige Voraussetzung für die Entwicklung einer guten Management-Poöitik. Daher wurden Struktur und Produktivität der Bestände von Acacia seyal und Acacia senegal in der natürlichen Vegetation von Massenya (Tschad) untersucht. Auf 32 Flächen von $900 \mathrm{~m}^{2}$ wurden Durchmesser und Höhe aller Gehölz-Individuen. Für Sträucher wurde der Durchmesser bei $50 \mathrm{~cm}$ vom Boden gemessen. Die jungen Exemplare wurden gezählt und Höhenklassen zugeordnet. Die Struktur der Population wurde durch die Anzahl der Stämme pro Hektar und die Durchmesserklassen gekennzeichnet. Der Index des Wertes der Bedeutung (L'Indice de Valeur d'Importance IVI) wurde verwendet, um die Prävalenz der Arten zu beurteilen. Die Standproduktivität an Gummi wurde anhand der durchschnittlichen Produktion des Baumes ausgewertet, die $250 \mathrm{~g}$ betrug. Die Bestände von Massenyas Acacia sind 
sehr dicht (619 \pm 269 Stämme / ha), haben aber aufgrund einer hohen Anzahl von Individuen mit kleinem Durchmesser (11,1 $\pm 2,2 \mathrm{~cm})$ einen geringen Basalbereich $\left(7,1 \pm 1,2 \mathrm{~m}^{2} / \mathrm{ha}\right)$. Im Allgemeinen handelt es sich hierbei um Strauchpopulationen (Höhe 5,2 $\pm 0,9 \mathrm{~m}$ ) mit einem guten Aufkommen von Jungwuchs (408 $\pm 267 \mathrm{Stämme} \mathrm{/} \mathrm{ha),} \mathrm{der} \mathrm{sich} \mathrm{natürlich} \mathrm{regenerieren}$ kann. Allerdings scheint Acacia seyal andere Standorte als Acacia senegal zu besiedeln. Von den sieben Arten, die im Untersuchungsgebiet vorherrschen, haben Acacia seyal und Acacia senegal das höchste IVI (79 und 55). Arten mit gutem Regenerationspotential sind Acacia seyal (64,58 $\pm 8,26$ juv./ha), Acacia senegal ( $58 \pm 10 \mathrm{juv}$./ha) und Guiera senegalensis (51 \pm 8 juv./ha). Die jährliche Gummiproduktion wird auf $56 \pm 6 \mathrm{~kg} /$ ha des brüchigen Gummis (Acacia seyal) und $41 \pm 5 \mathrm{~kg} / \mathrm{ha}$ Hartgummi (Acacia senegal) geschätzt.

Schlagworte: Acacia senegal, Acacia seyal, Gummi-Produktivität, sudano-sahelische Zone, Tschad

\section{INTRODUCTION}

Le Tchad, comme les autres pays sahéliens, connaît ces dernières décennies, une baisse générale de la pluviométrie (DGMN 2014, OSS 2015). Cette crise écologique récurrente se traduit par une nette modification des différents ensembles écologiques: le secteur sahélien devient saharo-sahélien et celui nord-soudanien devient simplement sahélien (MÉLOM et al. 2015, Thiombiano et al. 2012). Ces changements écologiques se traduisent par une régression continuelle de la densité et de la diversité spécifique des plantes ligneuses dans la zone sahélienne (CLAUde et al. 1991, GANABA et GuINKo 1995). Certaines espèces deviennent rares ou disparaissent complètement dans leur aire de distribution. Or, en milieu sahélien, les ressources végétales ligneuses ont une grande importance pour le pâturage, les usages domestiques et la protection des sols (GrouZIS 1988, Claude et al. 1991). En effet, celles-ci forment un tapis végétal pérenne qui favorise, entre autre, le développement des fourrages herbacés, la germination des semences et la reconstitution des peuplements ligneux pendant la saison pluvieuse en améliorant les microclimats et la fertilité du sol (BELSKY et Canham 1994, AKro et Grouzis 1996, Aкpo 1997).

Dans un contexte de changement climatique, la flore soudanienne est de plus en plus envahie par celle sahélienne (MÉLOM et al. 2015) et notamment par des espèces du genre Acacia pour la strate ligneuse. Au Tchad, sur les 16 espèces d'Acacia inventoriées (BRUNDU et CAMARDA 2013), Acacia senegal (L.) Willd. et Acacia seyal Del. sont assez bien distribuées dans la zone sahélo-soudanienne et jouent un rôle économique important pour le pays. En effet, grâce à ces deux espèces, le Tchad est classé comme deuxième pays exportateur de gomme arabique après le Soudan (MüLLer et OKoro 2004, Soloviev et al. 2009, Daniele et al. 2011, CNUCED 2016). La région du Chari-Baguirmi (Massenya), qui est l'une des zones productrices de la gomme arabique au Tchad, renferme d'importants peuplements naturels d'Acacia senegal et Acacia seyal (NEPAD/FAO 2005, MbaYngOne et MÉlom 2014). Au Tchad, si quelques études s'étaient intéressées à la structure des ligneux en général (Poilecot et al. 2008 et 2009, BÉCHIR et KABORÉZoungRANA 2012), on note très peu celles consacrées aux Acacia (Poilecot et al. 2006 et 2007, SAÏDI et Daget 2013, NGARYo et al. 2010 et 2015 et NGARNOUGBER et al. 2017) et moins encore sur leur potentiel de production. La plupart des estimations sur la capacité de production de gomme du Tchad se sont basées sur l'offre du marché (MüLLER et OKORo 2004, DANIELE et al. 2011). Or, une bonne évaluation de la capacité de production d'une ressource doit s'appuyer sur son potentiel réel sur le terrain. C'est pourquoi, nous voudrions par ce travail, fournir des données sur la structure et la productivité des peuplements à Acacia senegal et à Acacia seyal de Massenya. Des données structurales et de productivité serviront de base pour une politique d'aménagement des formations forestières et d'utilisation rationnelle des ressources ligneuses du Tchad.

\section{MATÉRIEL ET MÉTHOdes D'ÉTUde}

\subsection{Site d'étude}

La zone d'étude est située dans la région de Chari-Baguirmi (Chef-lieu Massenya), entre les latitudes $10^{\circ}$ et $12^{\circ}$ Nord et longitudes $16^{\circ}$ et $18^{\circ}$ Est, à $175 \mathrm{~km}$ au sud-est de N'Djaména (fig. 1). Du point de vue phytogéographique, elle appartient au domaine soudanien et au secteur soudanosahélien (White 1986, MéLom et al. 2015). La pluviométrie moyenne annuelle des quinze dernières années (1999-2013) est de $698 \mathrm{~mm}$ à la station météorologique de Massenya et $782 \mathrm{~mm}$ à Ba-Illi (DGMN 2014). La saison de pluie dure en moyenne six mois (mai à octobre) et les mois les plus humides sont juillet et août.

C'est une immense plaine rompue par des dépressions. Le réseau hydrographique est constitué des fleuves Chari, Logone, le Bahr Erguig ou Bambassa et de nombreuses mares temporaires.

Les sols sont ferrugineux tropicaux faiblement lessivés, à taches et à rares concrétions. Les sols hydromorphes et les vertisols se rencontrent dans les dépressions ou en bordure de dépressions faiblement inondées. Ce sont des sols à texture limono-argileuse à argilo-limoneuse, parfois sabloargileuse.

Du nord au sud de la zone d'étude, on note une prédominance des savanes armées (arbustives à arborées) à Acacia seyal et/ou $A$. senegal qui cèdent progressivement la place aux savanes inermes diverses ou forêts claires à Anogeissus leiocarpa (DC.) Guill. \&Perr. (MÉLOM et al. 2015, OSS 2015).

La population humaine est constituée majoritairement de Baguirmiens ou Barma, suivis des Haoussas et Saras; d'autres ethnies mineures comme les Peulhs, les Bornous et Ouaddaiens sont également représentées (INSEED 2009). Ces populations pratiquent à la fois l'agriculture et l'élevage extensifs, qui sont consommateurs de grands espaces et destructeurs de l'environnement. La collecte des gommes constitue aussi une activité secondaire, mais économiquement très rentable pour ces populations.

\subsection{Collecte de données}

Les peuplements à Acacia ayant servi pour les mesures dendrométriques représentent $61,77 \%$ de relevés phytosociolo- 


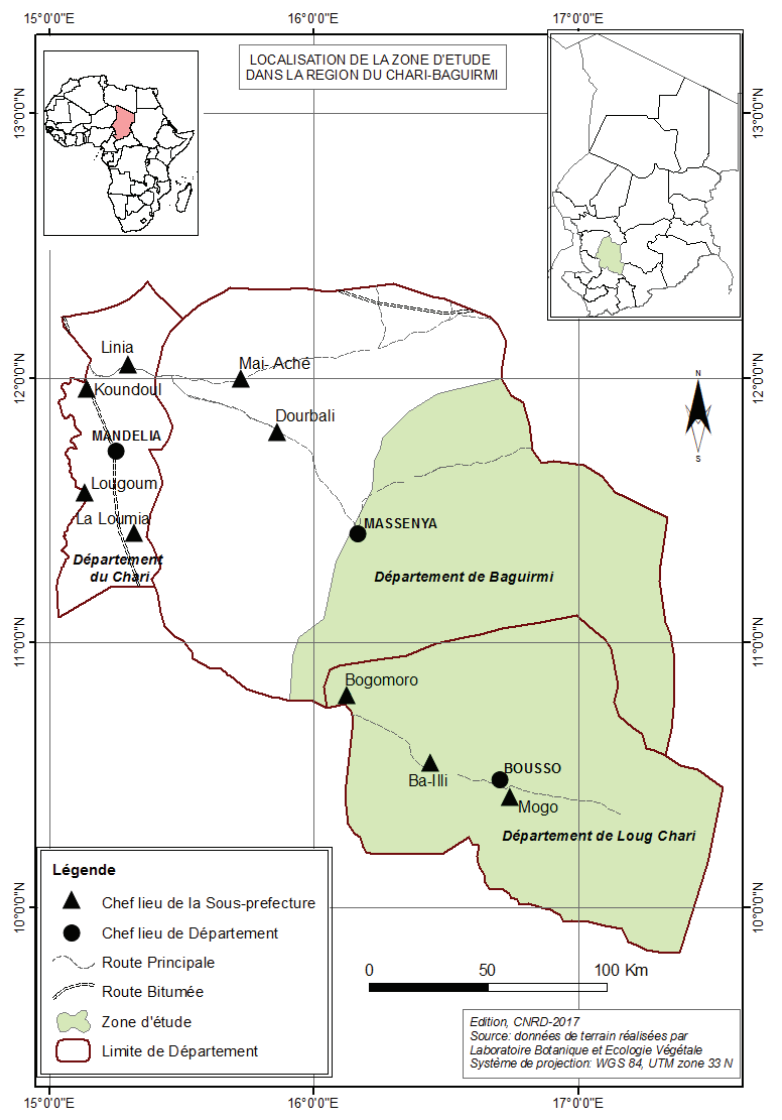

Fig. 1: Localisation de la zone d'étude. / Study zone location.

giques effectués dans la zone d'étude, soit 126 relevés sur 204, dont 46,57\% pour Acacia seyal et 15,20\% pour Acacia senegal (MéLom et al. 2015). Les peuplements à Acacia senegal colonisent généralement les plateaux dunaires ou non et les glacis divers, sur des sols de texture sableuse à sablo-limoneuse. Le plus souvent, Acacia senegal est l'espèce qui domine la strate arbustive. Les autres espèces ligneuses sont Ziziphus mauritiana Lam., Guiera senegalensis J.F.Gmel., Balanites aegyptiaca (L.) Delile, Acacia seyal, Dichrostachys cinerea (L.) Wight \&Arn et Sclerocarya birrea (A.Rich.) Hochst. Les espèces herbacées assez fréquentes sont Chloris pilosa Schumach., Cassia obtusifolia L., Zornia glochidiata Rchb. ex DC. et Triumfetta pentandra A.Rich. Les peuplements à Acacia seyal se rencontrent généralement dans les bas glacis, les axes de drainage et les bas-fonds inondables, sur des sols à texture sablo-limoneuse à argileuse. Ce sont des savanes arbustives, parfois arborées où Acacia seyal est parfois l'unique espèce ligneuse. Les rares espèces ligneuses qui complètent le cortège floristique sont les mêmes que celles des peuplements à Acacia senegal. La strate herbacée renferme le plus souvent Cassia obtusifolia L., Achyranthes aspera L., Panicum pansum Rendle, Andropogon gayanus Kunth, Chloris pilosa Schumach., Setaria pumila (Poir.) Roem. \& Schult., Monechma ciliatum (Jacq.) Milne-Redh., Pennisetum pedicellatum Trin. et Dinebra retroflexa (Vahl) Panz.
Pour chaque type de peuplement (Acacia senegal et Acacia seyal), seize (16) placeaux de $900 \mathrm{~m}^{2}$ ont été choisis au hasard pour des mesures dendrométriques. Ce sont les mêmes placeaux qui ont été utilisés lors des relevés phytosociologiques. Les paramètres mesurés sont la circonférence à 1,30 $\mathrm{m}$ et la hauteur totale des individus. Certains arbustes et arbrisseaux dont les individus adultes ne dépassent généralement pas $4 \mathrm{~m}$ sont mesurés à $50 \mathrm{~cm}$ du sol. Il s'agit de $B o-$ scia senegalensis, Flueggea virosa, Gardenia aqualla, Gardenia ternifolia, Ximenia americana, Feretia apodanthera, Calotropis procera, Dichrostachys cinera, Grewia bicolor, Grewia lasiodicus, Grewia villosa et Leptadenia hastata. En effet, le port arbustif de ces espèces leur confère beaucoup d'individus adultes de petit diamètre qui se retrouvent dans la population juvénile (Thiombiano 2005). Le choix de la circonférence permet de réduire au maximum les diverses sources d'erreur pouvant affecter l'estimation de la grosseur d'un arbre (Rondeux 1999). De ce fait, le diamètre est facilement déduit de la connaissance de circonférence mesurée à l'aide d'un ruban. Les individus qui ont une circonférence inférieure à $15,7 \mathrm{~cm}$ (ou diamètre $<5 \mathrm{~cm}$ ) sont classés dans la régénération. Ce sont de jeunes individus car nous avons tenu compte des plants susceptibles de résister à la pression du pâturage et à l'effet des feux de brousse. Pour les individus ramifiés, lorsque la ramification est en dessous de $1,3 \mathrm{~m}$, chaque branche est considérée comme un individu. Pour la mesure des hauteurs nous avons utilisé une perche de huit mètres $(8 \mathrm{~m})$ confectionnée à cet effet. Pour des individus très grands (plus de $10 \mathrm{~m}$ ) leur taille est estimée. Ces mesures ont été effectuées pendant les saisons sèches (novembre à mai) de 2011 à 2013.

\subsection{Traitement des données}

L'étude de la structure des peuplements a été réalisée à travers le nombre de tiges à l'hectare et les classes de diamètre. Un pas de cinq centimètres (MBAYNGONE et al. 2008) a été adopté pour les classes de diamètre afin d'apprécier la dynamique et les tendances évolutives de ces peuplements (Classe 1 ou classe des individus juvéniles $=<5 \mathrm{~cm}$, classe 2: $5-<10 \mathrm{~cm}$, classe $3: 10-<15 \mathrm{~cm}$, classe $4: 15-<20 \mathrm{~cm}$, classe 5: $20-<25 \mathrm{~cm}$, classe 6: $25-30 \mathrm{~cm}$, classe $7:>30 \mathrm{~cm}$. Les individus juvéniles ont été regroupés en cinq classes de hauteur $0-0,5 \mathrm{~m} ;>0,5-1 \mathrm{~m} ;>1-1,5 \mathrm{~m} ;>1,5-2 \mathrm{~m},>2 \mathrm{~m}$. Cette subdivision permet d'évaluer le potentiel de croissance et d'identifier les problèmes de développement des juvéniles.

Le test LSD de comparaison de moyenne a été effectué sous STATGRAPHICS Centurion XVI pour apprécier les différences des valeurs de densité, de surface terrière, diamètre et de hauteur au niveau des peuplements.

L'importance écologique des espèces a été appréciée à partir de l'Indice de Valeur d'Importance ou ImportanceValue Index (IVI) de Curtis \& Mcintosch (BANDA et al. 2006). Cet indice qui intègre la densité, la dominance et la fréquence permet de mieux apprécier l'importance d'une espèce dans une communauté végétale. 
Les caractéristiques structurales (densité de tiges, surface terrière, diamètre moyen) ont été calculées pour chaque placette et les moyennes ont été calculées par unité de végétation.

Les paramètres utilisés pour la caractérisation des peuplements ont été calculés de la manière suivante:

Dominance relative $=$ (surface terrière totale d'une espèce/ surface terrière totale de toutes les espèces) x 100

Densité relative $=$ (nombre d'individus d'une espèce/nombre total des individus) $\mathrm{x} 100$

Fréquence relative $=$ (fréquence d'une espèce/somme de toutes les fréquences) x 100

La productivité des peuplements en gomme arabique a été évaluée en prenant la valeur moyenne de production d'un arbre qui était de $250 \mathrm{~g}$ (http://unctad.org/fr/PublicationsLibrary/INFOCOMM_cp06_GumArabic_fr.pdf du 26 juillet 2016).

\section{RÉSULTATS}

\subsection{Structure des peuplements à Acacia seyal et $A$. sene- gal}

Au total 2958 tiges ont fait l'objet d'inventaire dans les 32 placeaux de $900 \mathrm{~m}^{2}$ échantillonnés $(2,88 \mathrm{ha})$, soit 1783 tiges d'individus adultes (diamètre $\geq 5 \mathrm{~cm}$ ) et 1175 tiges de population juvénile (individus $\leq 5 \mathrm{~cm}$ ). L'ensemble de ces individus appartient à 48 espèces et 38 genres répartis dans 17 familles dont les plus importantes sont les Fabaceae avec 13 espèces (Fabaceae-Mimosoideae 9, Fabaceae-Caesalpinioideae 3 et Fabaceae-Faboideae 1), les Combretaceae (7 espèces) et les Rubiaceae (6). Les Asclepiadaceae, les Capparaceae et les Rhamnaceae suivent avec 3 espèces chacune.

La densité moyenne des individus adultes est de $619 \pm 269$ tiges/ha pour une surface terrière de $7,1 \pm 1,2 \mathrm{~m}^{2} /$ ha, correspondant à un diamètre moyen de $11,1 \pm 2,2 \mathrm{~cm}$ pour une hauteur de 5,2 $\pm 0,9 \mathrm{~m}$. Ces différences sont significatives au seuil de 5\% (test LSD avec Ddl $=4 ; \mathrm{F}=144,14 ; \mathrm{p}=$ $0,0000)$, ce qui sous entend une grande variabilité des pa- ramètres structuraux en fonction des sites. Les peuplements à Acacia de la région du Chari-Baguirmi (Massenya) ont des densités assez fortes avec des individus de petit diamètre et une population juvénile bien représentée (fig. 2). Cette population juvénile a une densité moyenne de $408 \pm 267$ tiges/ha pour une hauteur de $2,3 \pm 0,5 \mathrm{~m}$.

La répartition de la population juvénile dans les cinq classes de hauteur retenues permet d'obtenir un histogramme en forme de «J» (fig. 3). Les classes des hauteurs les plus bassses ne présentent pas de différences significatives ( $\mathrm{Ddl}=5$; $\mathrm{F}=105,21$ et $\mathrm{p}=0,0000)$. Toutefois, la classe $>2 \mathrm{~m}$ est assez bien représentée et présente une différence significative avec toutes les autres classes.

Les espèces à bon potentiel de régénération sont Acacia seyal (65 \pm 9 juv./ha), Acacia senegal ( $58 \pm 11$ juv./ha) et Guiera senegalensis $(51 \pm 9$ juv./ha). Celles à potentiel de régénération moyenne sont Dichrostachys cinerea (29 \pm 5 juv./ha) et Dalbergia melanoxylon ( $23 \pm 4$ juv./ha). Les faible potentiels de régénération sont enregistrés chez Ziziphus mauritiana (18 \pm 3 juv./ha), Feretia apondonthera (17 \pm 3 juv./ha), Albizia chevalieri (13 \pm 4 juv./ha), Capparis sepiaria $(11 \pm 2 \mathrm{juv} . / \mathrm{ha})$ et Boscia senegalensis ( $10 \pm 2 \mathrm{juv}$./ha). Les autres espèces ont des potentiels de régénération très faibles, voire nul chez Sclerocarya birrea, Sterculia setigera et Acacia sieberiana.

De façon séparée, les peuplements à Acacia seyal sont les plus denses car ils renferment $712 \pm 151$ tiges/ha, pour une surface terrière moyenne de $4,0 \pm 0,9 \mathrm{~m}^{2} / \mathrm{ha}$, un diamètre de $10,9 \pm 1,2 \mathrm{~cm}$ et une hauteur moyenne de 5,6 $\pm 1,0 \mathrm{~m}$. Ces différences sont significatives pour les paramètres ci-dessus $(\mathrm{Ddl}=4 ; \mathrm{F}=303,15$ et $\mathrm{p}=0,000)$, traduisant ainsi la grande variabilité des sites $(n=16)$. Ce sont des peuplements relativement hauts avec généralement des individus de petit diamètre. Par contre, les peuplements à Acacia senegal sont moyennement denses avec $526 \pm 32$ tiges/ha, représentant une surface terrière moyenne de $3,13 \pm 0,7 \mathrm{~m}^{2} / \mathrm{ha}$, un diamètre moyen de 11,2 $\pm 2,8 \mathrm{~cm}$ et une hauteur de 4,8 $\pm 0,8 \mathrm{~m}$. Les différences observées sont significatives pour les quatre paramètres $(\mathrm{Ddl}=4 ; \mathrm{F}=35,56$ et $\mathrm{p}=0,000)$. Ces peuplements sont relativement bas avec une surface terrière faible par rapport à ceux d'Acacia seyal. La répartition des indi-

Tableau 1: Nombre de tiges à l'hectare par classes de diamètre dans les peuplements à Acacia seyal $(\mathbf{n}=16)$ et à $\mathrm{A}$. senegal $(\mathbf{n}=16)$. $/$ Table 1: Number of stems per hectare per diameter class in the Acacia seyal $(n=16)$ and A. senegal $(n=16)$ stands.

\begin{tabular}{|l|l|l|}
\hline Classes de diamètre & Peuplements à Acacia seyal & Peuplements à Acacia senegal \\
\hline$<5$ & $440,28 \pm 267,88 \mathrm{a}$ & $373,61 \pm 260,60 \mathrm{a}$ \\
\hline$[5-10]$ & $372,22 \pm 142,51 \mathrm{a}$ & $327,08 \pm 265,13 \mathrm{a}$ \\
\hline$[10-15]$ & $214,58 \pm 65,82 \mathrm{~b}$ & $131,94 \pm 86,95 \mathrm{~b}$ \\
\hline$[15-20]$ & $84,03 \pm 38,88 \mathrm{c}$ & $43,75 \pm 35,46 \mathrm{bc}$ \\
\hline$[20-25]$ & $30,56 \pm 24,06 \mathrm{c}$ & $10,42 \pm 11,43 \mathrm{c}$ \\
\hline$[25-30]$ & $7,64 \pm 10,18 \mathrm{c}$ & $2,08 \pm 4,34 \mathrm{c}$ \\
\hline$>30$ & $2,78 \pm 6,21 \mathrm{c}$ & $11,11 \pm 12,42 \mathrm{c}$ \\
\hline
\end{tabular}

Les valeurs qui portent la même lettre ne présentent pas de différences significatives entre les classes de diamètre; celles qui portent des lettres différentes ont des différences significatives. (Test LSD au seuil de 5\%). / The values which marked by the same letter dont present significative differences of means betwen class diameter; those were marked some different letters show significative differences (LSD test on the treshold of 5 per cent). 
vidus dans les différentes classes de diamètre est résumée dans le tableau 1 .

Dans les peuplements à Acacia seyal les deux classes les plus basses ne présentent pas de différences significatives, mais sont significativement différentes de la troisème classe. Les quatre autres classes supérieures ne présentent pas de différences significatives. Dans les peuplements à Acacia senegal, la même tendance se poursuit, sauf que la classe quatre présente des différences significatives avec les trois autres classes supérieures.

La distribution de la population des deux espèces (Acacia senegal et $A$. seyal) en classes de diamètre dans les peuplements permet d'évaluer leur amplitude écologique (fig. 4 et $5)$. En effet, chacune des deux espèces a une structure de population stable $(« L »)$ dans son peuplement respectif, même si on note une faible proportion des juvéniles par rapport à la classe $[5-<10]$. Ces faibles valeurs s'expliquent par le fait que les très jeunes plantules n'étaient pas prises en compte à cause de leur viabilité incertaine. On note une très faible re- présentativité de la population d'Acacia seyal dans les peuplements à Acacia senegal (fig. 4), bien qu'elle soit présente dans cinq sites de ces peuplements. La plupart des individus sont dans les classes $5-<10 \mathrm{~cm}$ et $10-<15 \mathrm{~cm}$ avec une régénération insignifiante.

Par contre, Acacia senegal, qui n'est présente que dans deux sites à Acacia seyal, a une structure de population stable dans ces peuplements même si les densités sont faibles (fig. 5).

Du point de vue importance des espèces, Acacia seyal et Acacia senegal prédominent sur les autres car leur Indices de Valeur d'Importance (IVI) sont respectivement 79,3 et 53,8 . Elles sont suivies de très loin par Piliostigma reticulatum $(17,0)$, Balanites aegyptiaca $(12,6)$, Sclerocarya birrea $(11,8)$, Dalbergia melanoxilon $(11,1)$ et Ziziphus mauritiana $(10,8)$. Ces sept espèces suscitées totalisent un IVI de 65,5 $\%$, ce qui sous-entend qu'elles influencent nettement la physionomie de la végétation de Massenya. La plupart des espèces ont des IVI très faibles (Tableau 2).


Fig. 2+3: Mode de distribution des individus dans les différentes classes de diamètre des peuplements à Acacia senegal et Acacia seyal $(\mathrm{n}=32)$. / Pattern of individuals distribution in the different classes of Acacia senegal and A. seyal stands ( $\mathrm{n}=32$ ).

Les histogrammes qui portent la même lettre ne marquent pas de différence significative, ceux qui ont les lettres différentes sont significativement différents. / The histograms which are marked by the same letter don not present significant differences of means between diameter classes, those marked with different letters weresignificantly different.
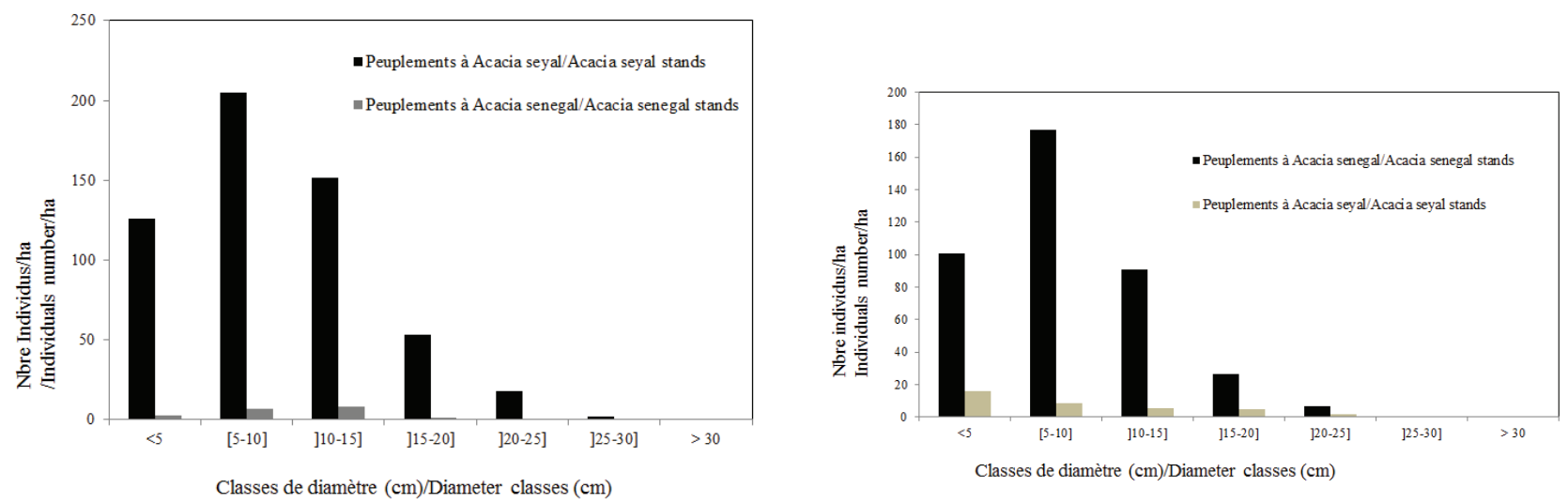

Fig. 4+5: Mode de distribution de Acacia seyal dans les peuplements à Acacia seyal $(\mathrm{n}=16)$ et à Acacia senegal $(\mathrm{n}=16)$. / Pattern of distribution of Acacia seyal in the Acacia seyal $(\mathrm{n}=16)$ and A. senegal $(\mathrm{n}=16)$ stands. 
Tableau 2: Récapitulatif des paramètres dendrométriques des espèces des peuplements à Acacia seyal et $A$. senegal $(\mathrm{n}=32$ relevés, avec Fr $=$ fréquence relative; $\mathrm{Dr}=$ densité relative; Dor $=$ dominance relative et IVI = indice de valeur d'importance). / Table 2: Summary of dendrometric parameters of species of Acacia seyal and $A$. senegal stands $(n=32$ relevés,with Fr $=$ relative frequency; Dr $=$ relative density; Dor $=$ relative dominance and $I V I=$ Importance Value index).

\begin{tabular}{|c|c|c|c|c|c|}
\hline Espèces & Fr & Dr & Dor & IVI & Familles \\
\hline Acacia seyal Del. & 8,98 & 36,01 & 34,34 & 79,33 & Fabaceae-Mimosoideae \\
\hline Acacia senegal (L.) Willd. & 5,47 & 26,19 & 22,14 & 53,80 & Fabaceae-Mimosoideae \\
\hline Piliostigma reticulatum (DC.) Hochst. & 5,47 & 6,45 & 5,06 & 16,98 & Fabaceae-Caesalpinioideae \\
\hline Balanites aegyptiaca (L.) Del. & 4,69 & 2,92 & 5,03 & 12,64 & Zygophyllaceae \\
\hline Sclerocarya birrea (A.Rich.) Hochst. & 1,17 & 1,01 & 9,64 & 11,82 & Anacardiaceae \\
\hline Dalbergia melanoxylon Guill. \& Perr. & 6,25 & 2,75 & 2,12 & 11,12 & Fabaceae-Faboideae \\
\hline Ziziphus mauritiana Lam. & 5,47 & 3,42 & 1,92 & 10,81 & Rhamnaceae \\
\hline Albizia chevalieri Harms & 1,17 & 3,93 & 3,54 & 8,64 & Fabaceae-Mimosoideae \\
\hline Commiphora pedunculata (Kotsch. \& Peyr.) Engl. & 3,52 & 2,58 & 2,45 & 8,54 & Burseraceae \\
\hline Acacia amythethophylla Steud.ex A. Rich. & 2,73 & 1,91 & 3,14 & 7,78 & Fabaceae-Mimosoideae \\
\hline Dichrostachys cinerea (L.) Wight \& Arn & 5,47 & 1,07 & 0,32 & 6,85 & Fabaceae-Mimosoideae \\
\hline Anogeissus leiocarpa (DC.) Guill. \& Perr. & 1,95 & 2,02 & 2,63 & 6,61 & Combretaceae \\
\hline Combretum glutinosum Perr. ex DC. & 3,52 & 1,46 & 1,16 & 6,14 & Combretaceae \\
\hline Capparis sepiaria $\mathrm{L}$. & 5,47 & 0,50 & 0,12 & 6,10 & Capparaceae \\
\hline Guiera senegalensis J.F.Gmel. & 5,08 & 0,79 & 0,23 & 6,09 & Combretaceae \\
\hline Mitragyna inermis (Willd.) Kuntze & 2,73 & 1,57 & 0,88 & 5,18 & Rubiaceae \\
\hline Feretia apodanthera Delile & 4,30 & 0,67 & 0,18 & 5,15 & Rubiaceae \\
\hline Sterculia setigera Delile & 0,78 & 0,39 & 2,41 & 3,58 & Malvaceae \\
\hline Grewia lasiodiscus K.Schum. & 3,13 & 0,00 & 0,00 & 3,13 & Malvaceae \\
\hline Boscia senegalensis (Pers.) Lam. ex Poir. & 2,73 & 0,11 & 0,03 & 2,87 & Capparaceae \\
\hline Flueggea virosa (Roxb. ex Willd.) Voigt & 2,73 & 0,00 & 0,00 & 2,73 & Euphorbiaceae \\
\hline Terminalia avicennioides Guill. \& Perr. & 0,39 & 1,18 & 0,85 & 2,42 & Combretaceae \\
\hline Cassia sieberiana DC. & 1,95 & 0,17 & 0,21 & 2,33 & Fabaceae-Caesalpinioideae \\
\hline Acacia ataxacantha DC. & 1,95 & 0,00 & 0,00 & 1,95 & Fabaceae-Mimosoideae \\
\hline Combretum aculeatum Vent. & 1,56 & 0,06 & 0,04 & 1,66 & Combretaceae \\
\hline Diospyros mespiliformis Hochst. ex A.DC. & 0,39 & 0,39 & 0,47 & 1,25 & Ebenaceae \\
\hline Cissus quadrangularis L. & 1,17 & 0,00 & 0,00 & 1,17 & Vitaceae \\
\hline Acacia sieberiana $\mathrm{DC}$. & 0,39 & 0,23 & 0,55 & 1,17 & Fabaceae-Mimosoideae \\
\hline Gardenia aqualla Stapf \& Hutch. & 0,39 & 0,72 & 0,00 & 1,11 & Rubiaceae \\
\hline Catunaregam nilotica (Stapf) Tirveng. & 0,39 & 0,45 & 0,16 & 1,00 & Rubiaceae \\
\hline Combretum nigricans Lepr. ex. Guill. \& Perr. & 0,78 & 0,11 & 0,07 & 0,96 & Combretaceae \\
\hline Stereospermum kunthianum Cham. & 0,78 & 0,06 & 0,03 & 0,87 & Bignoniaceae \\
\hline Cadaba benguellensis Mendes & 0,78 & 0,06 & 0,01 & 0,85 & Capparaceae \\
\hline Annona senegalensis Pers. & 0,39 & 0,34 & 0,11 & 0,84 & Annonaceae \\
\hline Tamarindus indica $\mathrm{L}$. & 0,78 & 0,00 & 0,00 & 0,78 & Fabaceae-Caesalpinioideae \\
\hline Acacia nilotica (L.) Willd. ex Del. & 0,39 & 0,22 & 0,00 & 0,61 & Fabaceae-Mimosoideae \\
\hline Rytigynia senegalensis Blume & 0,39 & 0,06 & 0,07 & 0,52 & Rubiaceae \\
\hline Ziziphus mucronata Willd. & 0,39 & 0,06 & 0,04 & 0,49 & Rhamnaceae \\
\hline Lannea barteri (Oliv.) Engl. & 0,39 & 0,06 & 0,02 & 0,47 & Anacardiaceae \\
\hline Calotropis procera (Ait.) Ait. f. & 0,39 & 0,06 & 0,01 & 0,46 & Asclepiadaceae \\
\hline Hyphaene thebaica (L.) Mart. & 0,39 & 0,06 & 0,01 & 0,46 & Arecaceae \\
\hline Gymnema sylvestre (Retz.) Schult. & 0,39 & 0,00 & 0,00 & 0,39 & Asclepiadaceae \\
\hline Leptadenia hastata (Pers.) Decne. & 0,39 & 0,00 & 0,00 & 0,39 & Asclepiadaceae \\
\hline Terminalia mollis M.A.Lawson & 0,39 & 0,00 & 0,00 & 0,39 & Combretaceae \\
\hline Entada africana Guill. \& Perr. & 0,39 & 0,00 & 0,00 & 0,39 & Fabaceae-Mimosoideae \\
\hline Ximenia americana $\mathrm{L}$. & 0,39 & 0,00 & 0,00 & 0,39 & Olacaceae \\
\hline Ziziphus abyssinica A.Rich. & 0,39 & 0,00 & 0,00 & 0,39 & Rhamnaceae \\
\hline Gardenia ternifolia Schumach. \& Thonn. & 0,39 & 0,00 & 0,00 & 0,39 & Rubiaceae \\
\hline Total & 100,00 & 100,00 & 100,00 & 300,00 & \\
\hline
\end{tabular}




\subsection{Productivité de Acacia seyal et $\boldsymbol{A}$. senegal}

Sur les $619 \pm 269$ tiges/ha, Acacia seyal et A. senegal renferment les densités les plus fortes, avec respectivement 223 \pm 23 tiges/ha et $162 \pm 22$ tiges/ha. Ce qui donnera une production moyenne de $56 \pm 6 \mathrm{~kg} / \mathrm{ha}$ de gomme friable (à Acacia seyal) et $41 \pm 5 \mathrm{~kg} / \mathrm{ha}$ de gomme dure (à $A$. senegal), soit une production globale de $96 \pm 12 \mathrm{~kg} / \mathrm{ha}$ de gomme dans la zone d'etude en zone soudano-sahélienne du Tchad.

\section{Discussion}

\subsection{Structure des peuplements à Acacia seyal et à Acacia senegal}

Les peuplements à Acacia de Massenya présentent une richesse spécifique plus élevée (48 espèces) que ceux définis dans les régions nord-soudaniennes à soudaniennes (MbaYngone 2008, Poilecot et al. 2006 et 2009, NACoulMA 2012). Ces auteurs ont obtenu des richesses spécifiques de 25 à 35 espèces dans les groupements à Acacia. Ces faibles valeurs traduiraient les conditions climatiques plus favorables au développement des Combretaceae, car les pluviométries moyennes annuelles sont supérieures (800 et 1000 $\mathrm{mm})$ à celles de notre site d'étude $(600$ à $700 \mathrm{~mm})$. La xéricité du milieu d'étude est caractéristique du secteur sahélosoudanien qui est le domaine des espèces épineuses, notamment des Acacia (White 1986, Arbonnier 2002, WitTig et al. 2004, Thiombiano et al. 2012). Ce qui est d'ailleurs confirmé par la prédominance des Fabaceae-Mimosoideae sur les autres familles.

Les plus fortes valeurs de densité des individus adultes obtenues $(619 \pm 269$ tiges/ha) sont influencées par la population d'Acacia seyal qui colonise plusieurs milieux et à une distribution grégaire. En effet, MBAYNGONE et al. (2008) ont relevé que le mode de distribution et la plasticité écologique de certaines espèces influent fortement sur la densité des individus. Acacia seyal, qui apparait souvent en peuplements purs sur divers milieux, présente une densité assez forte dans ses peuplements naturels (Sop et al. 2010). Les faibles valeurs des surfaces terrières et des diamètres traduisent l'abondance des individus de faible grosseur dans les peuplements à Acacia de Massenya. En effet, les peuplements savanicoles sahélo-soudaniens présentent souvent des individus de petite grosseur et relativement bas (MBAYNGONE et al. 2008, Poilecot et al. 2009, Ngaryo et al. 2010, SCHMIDT et al., 2013, NGARNOUGBER et al. 2017) car, même, en milieu soudanien, donc plus humide (pluviométrie 923,12 mm/an), BÉCHIR et KABORÉ-ZoungranA (2012) ont obtenu des valeurs plus petites de diamètre $(8,8 \pm 0,5 \mathrm{~cm})$ aux nôtres. Ce constat mérite d'être développé afin de revoir en baisse la limite de $5 \mathrm{~cm}$ admise généralement pour les individus adultes en zone sahélo-soudanienne. Cela permettrait de révéler les densités réelles des espèces ligneuses de cette zone bioclimatique.

La répartition des individus en classes de diamètre, qui présente une structure démographique en forme de $\langle L »$, traduit des formations stables (OuÉDraOGo et al.2006). Cette structure, marquée par une réduction régulière du nombre d'individus d'une classe de dimension à la suivante, caractérise celle de peuplements capables de recruter des indivi- dus dans le temps, donc susceptibles de se régénérer naturellement (MBAYNGONE et al. 2008, Thiombiano et al. 2010, SAMBARÉ et al. 2011). De telles distributions révèlent donc un bon potentiel de régénération de la population étudiée. Ce qui est confirmée par la distribution de la population juvénile en classe de hauteur qui ne présente pas de différence significative. Les faibles proportions des juvéniles observées dans les deux classes les plus basses sont dues au fait que ces classes sont soumises à diverses intempéries tels que le broutage des petits ruminants, le surpâturage, le piétinement des bovins et l'effet des feux de brousse (Sop et al. 2010). Ces contraintes traduisent la difficulté de la population juvénile à recruter dans les deux classes de hauteurs les plus basses. Mais comme les espèces à Acacia, surtout Acacia seyal, ont une croissance rapide en saison de pluie (Poilecot et al. 2007), nous observons une bonne représentation des juvéniles dans les classes 1-1,5 à $>2 \mathrm{~m}$, pouvant assurer le renouvellement naturel des peuplements étudiés. Les peuplements à Acacia senegal et A. seyal sont donc des peuplements viables car la population juvénile est bien représentée pour assurer leur renouvellement naturel.

Dans ces peuplements, Acacia seyal, Acacia senegal et Guiera senegalensis feront toujours partie du cortège floristique dominant car elles ont un bon potentiel de régénération. Par contre, une attention particulière doit être accordée à Sclerocarya birrea, Sterculia setigera et Acacia sieberiana qui ne présentent pas de population juvénile. En effet, les deux premières espèces présentent toujours des difficultés de régénération dans les savanes soudaniennes à soudanosahéliennes (MBAYNGONE et al. 2008, BÉCHIR et KABORÉZoungrana 2012) alors que Acacia sieberiana supporte moins bien les inondations que Acacia seyal (PoILecot et al. 2009). Les inondations prolongées constituent une contrainte majeure à la survie des plantules d'Acacia sieberia$n a$.

En considérant séparément les deux peuplements, Acacia seyal présente une grande amplitude écologique par rapport à Acacia senegal car elle colonise au moins cinq de ses peuplements. En effet, Acacia seyal se développe sur les bas de pentes et bas-fonds, sur sols argileux et lourds ou sur sols pierreux et supporte les inondations temporaires suivies de sécheresses périodiques (ARbonNier 2002, Poilecot et al. 2006, Sop et al. 2010). Ce qui lui offre une grande facilité de conquérir beaucoup de milieux contrairement à Acacia senegal qui préfère les sols sableux, même si l'on peut la rencontre occasionnellement sur les sols limoneux légers à brun argileux (ARBONNIER 2002). Ces résultats montrent que, dans les formations naturelles de Massenya, Acacia seyal est plus apte à coloniser d'autres milieux que son milieu de prédilection qui renferme généralement ses peuplements. Par contre, Acacia senegal, même si elle est confinée dans ses peuplements, présente toujours une structure de population stable dans ceux à Acacia seyal colonisés.

Du point de vue physionomique, les peuplements à Acacia de Massenya seront marqués par la prédominance de sept espèces, à savoir Acacia seyal, Acacia senegal, Piliostigma reticulatum, Balanites aegyptiaca, Sclerocarya birrea, Dalbergia melanoxilon et Ziziphus mauritiana. Ces espèces réalisent $65,50 \%$ des Indices de Valeur d'Importance de l'ensemble. De telle prédominance des formations natu- 
relles par un petit nombre d'espèces, accompagnées par un grand nombre d'espèces rares, a été mise en évidence par certains auteurs (Wala 2004, Bognounou et al. 2009). Parmi ces espèces, certaines s'imposent par l'importance de leur densité et dominance relatives (cas d'Acacia seyal et Acacia senegal), tandis que d'autres, seulement par la dominance relative Sclerocarya birrea). En effet, Sclerocarya birrea qui ne présente que des individus arborés sur le site d'étude, a une dominance relative importante $(9,64)$ à cause de la grosseur de ses sujets adultes.

\subsection{Productivité d'Acacia seyal et Acacia senegal}

La production de gomme estimée ici constitue un indicateur d'incitation pour les investisseurs de la filière. Cette valeur pourrait augmenter encore significativement si l'on considère l'aire totale de distribution des deux espèces au Tchad qui étaiet estimée à 30 millions d'hectare (NEPAD/ FAO 2005). En effet, Acacia seyal et A. senegal sont mieux répandues dans les zones à pluviométrie faibles (ARBONNIER 2002, Traoré et al., 2008, Soloviev et al. 2009). Au Tchad, les meilleurs peuplements d'Acacia seyal et $A$. senegal sont répertoriés dans les régions du sahel (Guéra (Centre Sud), Batha (Centre Nord), Ouaddaï et Wadi Fira (Est)) et le nordsoudanien (Chari-Baguirmi (Ouest)) (NGARYo et al. 2015). Aussi, des valeurs de production de gomme plus élevées (entre 20 et $2000 \mathrm{~g}$ par sujet) ont été révélées, mais la moyenne généralement admise est de 250g/arbre (CNUCED 2016). Tout porte à croire que si nous réalisons un inventaire exhaustif des peuplements à Acacia producteurs de gomme, l'offre tchadienne de ce produit pourrait considérablement augmenter. Cela constituerait une source de revenu non négligeable pour la population rurale car la gomme représentait le troisième produit d'exportation du Tchad avant l'ère pétrolière (Muller et Okoro 2004).

\section{Conclusion}

Les peuplements à Acacia de Massenya présentent une richesse spécifique assez élevée (48 espèces), malgré une tendance de distribution septentrionale (milieux plus secs) des deux espèces. Ce sont des peuplements assez denses, dominés par des individus de petit diamètre. Ces peuplements sont stables, susceptibles de se régénérer d'eux-mêmes, car ils disposent d'un bon potentiel de régénération. Toutefois, l'absence de régénération observée chez certaines espèces pourrait se traduire par des conditions spécifiques de milieux qui abritent ces peuplements. Qu'à cela ne tienne, ce sont des peuplements dont la physionomie sera fortement influencée par quelques espèces dominantes comme Acacia seyal, Acacia senegal, Piliostigma reticulatum, Balanites aegyptiaca, Sclerocarya birrea, Dalbergia melanoxilon et Ziziphus mauritiana qui ont des indices de Valeur d'importance élevés. La productivité de gomme des espèces évaluée constitue une piste de recherche pour l'amélioration globale de l'offre tchadienne en gomme arabique d'une part, et d'autre une source de revenus pour la population locale.

\section{REMERCIEMENTS}

Nous remercions vivement l'Université de N'Djaména qui a financé ces travaux de recherche à travers le fonds d'appui à la recherche. Nous remercions également les autorités décentralisées du Ministère de l'Intérieur et de l'Environnement des Départements du
Baguirmi et de Loug-Chari qui nous ont apporté le soutien nécessaire lors de la collecte des données. Aux référés anonymes qui ont lu avec beaucoup d'attention et fait des commentaires très utiles au manuscrit, nous adressons nos sincères gratitudes.

\section{REFERENCES}

AKPo L-E (1997): Phenological interactions between tree and understory herbaceous vegetation ofa sahelian semi-arid savanna. Plant Ecol 131: 241-248.

Akpo L-E \& Grouzis M (1996): Observations phénologiques en milieu sahélien: éléments d'interprétation des relations herbe/arbre. An. Univ. Ouagadougou. Série B, Vol. IV: 369-380.

Arbonnier M (2002): Arbres, arbustes et lianes d'Afrique de l'Ouest. 2eme edition, CIRAD-MNHN- UICN, 541 p.

BANDA T, SchWARTz M W \& CARo T (2006): Woody vegetation structure and composition along a protection gradient in a Miombo ecosystem of western Tanzania. - Forest Ecol Management, 230: 179-185.

BÉChIR A B \& Kabore-Zoungrana C (2012): Fourrages ligneux des savanes du Tchad: structure démographique et exploitations pastorales. Cameroon J Experimental Biol 8: 35-46.

Belsky A J \& CANHAM C D (1994): Forest gaps and isolated savanna trees. An application of patch dynamics in two ecosystems. BioSciee 44: 77-84.

Bognounou F, Thiombiano A, Savadogo P, Boussim J I, ODEN P C \& GUINKo S (2009): Woody vegetation structure and composition at four sites along a latitudinal gradient in Western Burkina Faso. Bois Forêts Tropiques 300 (2): 29-44

BRUNDU G \& CAMARDA I (2013): The flora of Chad a checklist and brief analysis. PhytoKeys 23: 1-17.

Claude J, Grouzis M \& Milleville P (1991): Un espace sahélien. La mare d'Oursi. Burkina Faso. Ed. ORSTOM, Montpellier, France, 241p.

CNUCED 2016: Gomme arabique. Nations Unies/CNUCED, 15p. [http://unctad.org/fr/PublicationsLibrary/INFOCOMM_cp06_GumArabic_fr.pdf du 26 juillet 2016].

Daniele C, El Asmar T \& Riccioli F (2011): Analyse des potentialités de la commercialisation de la gomme arabique (Acacia senegal) sur les marchés Italiens et Européens. J. Agr. Envir. Int. Dev. 105: 3-24.

DREM 2014: Les données climatologiques de Massenya et Ba-Illide1999 à 2013.Ministère Aviation Civile et Météorologie Nationale.

Ganaba S \& Guinko S (1995): Etat actuel et dynamique du peuplement ligneux de la région de la mare d'Oursi (Burkina Faso). Etudes flor vég Burkina Faso 2: 3-14.

Grouzis M (1988): Structure, productivité et dynamique des écosystèmes écologiques sahéliens (mare d'Oursi, Burkina Faso). Doctorat d'Etat ès-Sciences Naturelles, Univ. Paris Sud, ORSTOM, 336p.

INSEED (2009): Deuxièmerecensement général de la population et de l'habitat. Ministère de l'économie et du plan, 89p.

MbaYngOne E (2008): Flore et végétation de la réserve partielle de faune de Pama, sud-est du Burkina Faso. Doctorat Univ. Ouagadougou, 181p +Annexes.

Mbayngone E, Thiombiano A, Hahn-Hadjali K \& GuinKO S (2008): Structure des ligneux des formationsvégétales 
de la Réserve de Pama (Sud-Est du Burkina Faso, Afrique de l'Ouest). Flora Veg Sudano-Sambesica 11: 25-34.

MbaYngone E \& MÉLom S (2014): Les savanes de la zone sahélo-soudanienne du Tchad: Phytocoenoses et Phytodiversite des formations naturelles de Massenya. Projet de recherche, rapport final. Université de N'Djaména, 37p.

Mélom S, Mbayngone E, Béchir A B, Ratnan N \& Mapongmetsem P-M (2015): Caractéristiques floristique et écologique des formations végétales de Massenya au Tchad (Afrique centrale). J Animal Plant Sci 25: 3799 -3813.

MÜLler D \& OKORo C (2004): Production et commercialisation de la gomme arabique. NGARA 2: 44-88.

Nacoulma B (2012): Dynamique et stratégie de conservation de la végétation et de la phytodiversté du complexe écologique du Parc National du W du Burkina-Faso. Thèse Unique, Univ. Ouagadougou. 153p + Annexes.

Ngarnougber C, Ngaryo F T \& Adamou I (2017): Caractérisation des ligneux de la savane sahélienne à Acacia senegal (L.) Willd dans la région du Guéra, Tchad. Int J Appl Research 3: 600-606.

NEPAD/FAO (2005): Appui à la mise en oeuvre du NEPAD-PDDAA, Volume IV de IV. Développement de la filière gomme arabique du Tchad, 26p.

Ngaryo F T, Goudiaby V \& AKro L E (2010): Characteristics of a plantation of Acacia senegal (L.) Willd. in the region of the Chari-Baguirmi in Chad. J des Sciences 10(2): 13-23.

Ngaryo F T, Adey S A, Guessolta E \& Goudiaby V (2015): Tapping method and management of Acacia senegal (L.) Willd. in a sahelian savannah of Chad. Int J Sci Adv Tech. 5(5): 7-13.

OSS (2015): Tchad: Atlas des cartes de végétation - Projet amélioration dela résilience des populations sahéliennes aux mutations environnementales (REPSAHEL).

Oú́draogo A, Thiombiano A, Hahn-Hadjali K \& GuinKO S (2006): Diagnostic de l'état dedégradation des peuplements de quatreespèces ligneuses en zone soudanienne du Burkina Faso. Sécheresse 17: 485-491.

Poilecot P, Boulanodji E, Taloua N, Dimet B, Ngui T\& Singa J (2006): Parc National de Zakouma: Structure des peuplements ligneuxdans des savanes exploitées par les éléphants. Bois Forets Tropiques 290(4): 45-59.

Poilecot P, Boulanodji E, Taloua N, Dismet B, Ngui T\& Singa J (2007): Parc national de Zakouma: des éléphants et des arbres. Bois et Forets des Tropiques 291(1): 13-24.

Poilecot P, Daget P\& N'Gakoutou E B (2008): Sur la structure spatiale d'une savane à Combretaceae dans les sud-est du Tchad. Acta Bot. Gallica, 155: 335-340.

Poilecot P , Saïdi S \& N'Gakoutou E B (2009): Phytogéographie du Parc national de Zakouma (Sud-Est du Tchad). Sécheresse 20: 286-295.

RONDEUX J (1999): La mesure des arbres et des peuplements forestiers. 2ème édition, Presses Agronomiques Gembloux, Belgique, 520p.

SAÏDI S \& DAGET P (2013): Stratégie de pâturage d'une population d'éléphants en saison sèche: le cas du parc de Zakoumaau Tchad. Bois et Forets des Tropiques 317(3): 59-70.

Sambaré O, Bognounou F, Wittig R \& Thiombiano A (2011): Woody species composition, diversity and structure ofriparian forests of four water courses types in Burkina Faso. J Forestry Research 22: 145-158.

Schmidt M, Traoré S, Ouédraogo A, Mbayngone E, OuÉdraogo O, ZizKa A, Kirchmair I, Kaboré E, Tindano E, Thiombiano A, Hahn K \& ZizKa G (2013): Geographical patterns of woody plants' functional traits in Burkina Faso. Candollea 68: 197-207.

Soloviev P, Zerbo G C, Lompo D, Yoda L B, Jaceues D \& Diallo A (2009): Acacia senegal au Burkina Faso: État de la ressource et potentiel productif. Bois Forêts Tropiques 300(2): 15-25.

Sop T K, Oldenland J, Schmidel U, Ouédraogo I \& ThIOMBIANO A (2010): Population structure of three woody species in four ethnic domains of the Sub-sahel of Burkina Faso. Land Degrad. Develop.

Thiombiano A (2005). Les Combretaceae du Burkina-Faso: taxonomie, écologie, dynamique et régénération des espèces. Thèse d'Etat, Univ. Ouagadougou. 290 pages + annexes.

Thiombiano A, Schmidt M, Dressler S, OuÉdraogo A \& ZizKA G (2012): Catalogue des plantes vasculaires du Burkina Faso. Boissiera 65, 391p.

Thiombiano D N E, Lamien N, Dibong S D, Boussim I J (2010): Etat des peuplements des espèces ligneuses desoudure des communes rurales de Pobé-Mengaoet de Nobéré (Burkina Faso). Journal Animal Plant Sci 9: 1104- 1116.

Traoré S, Kaboré O, Thiombiano L \& RasolodimbyMillogo J (2008): Prédiction spatiale et modélisation de la distribution des Acacia ssp. Dans l'Est du Burkina Faso. Sécheresse 19: 283-92.

WALA K (2004): La végétation de la chaîne de l'Atakora au Bénin: diversité floristique, phytosociologie et impact humain. Thèse de Doctorat de l'Université de Lomé, 140 p.

White F (1986): La végétation de l'Afrique: mémoire accompagnant la carte devégétation de l'Afrique. Paris, ORSTOM, 384 p.

Wittig R, Schmidt M. Thiombiano A (2004): Cartes de distribution des espèces du genres Acacia L. au Burkina Faso. Etudes flor vég Burkina Faso 8: 19-26. 